\title{
Electrochemical Reactions of Sodium 2-Ethylhexyl Sulfate Salt
}

\author{
Aleksandra Perek-Dlugosz ${ }^{1}$ - Adam Socha ${ }^{1} \cdot$ Jacek Rynkowski $^{1}$
}

Published online: 1 March 2017

(C) The Author(s) 2017. This article is published with open access at Springerlink.com

\begin{abstract}
The electrochemical reactions of sodium 2-ethylhexyl sulfate (EHS) and its effect on the $\mathrm{Zn}^{2+}$ electroreduction have been investigated at a mercury electrode using cyclic voltammetry. It has been shown that the reduction takes place in two steps. The presence of EHS in the solution containing $\mathrm{Zn}^{2+}$ ions moves slightly the potential of zinc reduction towards more negative potentials and causes a slight increase in current density. The differential capacity-potential and differential capacity-time measurements indicate strong adsorption in a wide potential range on the electrode surface. In the potential range -0.46 to $-0.86 \mathrm{~V}$ vs. saturated calomel electrode and at the concentration lower than the critical micelle concentration (CMC), adsorption for the longer time is hardly reversible. At the concentration higher than the $\mathrm{CMC}$, the formation of hemispherical surface micelles is observed. The theoretical maximum degree of electrode coverage computed with the use of quantum-chemical calculations is equal to $3.53 \times 10^{14}$ particles $\mathrm{cm}^{-2}$, and it is larger than the value determined experimentally from cyclic voltammograms. In the case of electrochemical reaction, at a current of $0.3 \mathrm{~A}$, during $180 \mathrm{~min}$, the obtained mineralization of EHS is only $3 \%$.
\end{abstract}

Keywords Adsorption - Electroreduction - Mineralization · Sodium 2-ethylhexyl sulfate

Aleksandra Perek-Dlugosz aleksandra.perek-dlugosz@o2.pl

1 Faculty of Chemistry, Institute of General and Ecological Chemistry, Lodz University of Technology, ul. Zeromskiego 116, 90-924 Lodz, Poland

\section{Introduction}

The electrodeposits of zinc are considered to be one of the main methods enabling anticorrosive protection of steel. Such properties can be achieved by adding certain organic compounds, i.e., arenes and surface-active agents to the zinc bath $[1,2]$. Surfactants are commonly used in zinc electrodeposition to control both the shape and size of metallic crystals and, in consequence, to obtain smooth and bright coatings [3, 4]. Solubilization of organic compounds in the presence of their aggregates is also a well-known phenomenon. The most often used surfactants may be classified as ionic (anionic, cationic, zwitterionic) and non-ionic [5]. Anionic surfactants with a negatively charged head group and a positively charged counterion, mostly sodium, have many applications, i.e., in galvanic industry. Their specific activity during electrodeposition depends on their concentration and molecule adsorption on the cathode surface [6]. An example in this group of surfactants is 2-ethylhexyl sulfate (EHS), a commonly used additive in electroplating baths that allows dissolution of sparingly soluble substances in water.

At low concentrations, surfactant molecules exist in an aqueous medium in the form of solvated monomers. However, above a particular concentration, known as the critical micelle concentration (CMC), they exhibit an ability to form self-aggregated structures - micelles. Then, their separated water hydrophobic tails aggregate into a hydrophobic interior with a hydrophilic surface [7]. The CMC depends on many factors such as temperature, ionic strength, surfactant chemistry, and the presence of other organic additives in the solution $[8,9]$.

The use of different surfactants may result in different effects in the process of metal deposition. The accelerating effect of an anionic surfactant on $\mathrm{Zn}^{2+}$ electroreduction was observed in the work of Nieszporek [10] and Gomes and da Silva Pereira [3]. The measurements performed at the dropping mercury electrode showed a current increase without the change in the peak 
potential with the addition of 1-decanesulfonate to the zinc solution. Opposite results were obtained in the presence of another anionic alkyl surfactant such as sodium dodecyl sulfate (SDS) [3, 4], for which the authors proved a negative shift with its addition. The process of zinc electroreduction at electrode materials such as $\mathrm{Pt}, \mathrm{Pd}, \mathrm{Au}$, and steel is characterized by underpotential deposition (UPD) and bulk deposition [11-14]. The UPD process at steel surface starts at the potential of $-0.85 \mathrm{~V}$ vs. saturated calomel electrode (SCE), and the maximum current is reached at $-1.05 \mathrm{~V}$ vs. SCE [13]. Simultaneously, hydrogen evolution takes place $[13,14]$. The effect of cationic (cetyltrimethylammonium bromide, CTAB), anionic (SDS), and non-ionic ( $N$-dodecyl- $N, N$-dimethyl-3-ammonio-1-propanesulfonate, Triton X-100) surfactants on $\mathrm{Zn}$ electrodeposition was investigated by Gomes and da Silva Pereira [13]. They found a distinct effect of CTAB and Triton X-100 addition on the reaction of zinc bulk deposition whereas the presence of SDS does not cause significant changes. $\mathrm{Zn}$ deposits prepared in the absence of surfactants and in the presence of SDS are more crystalline and have a higher grain size than the ones obtained in the presence of CTAB and Triton X-100.

The mass of detergents adsorbed on the surface of various electrode materials grows practically linearly in relation to the $\mathrm{c} / \mathrm{cCMC}$ ratio equal to 1 . Further increase in concentration does not increase the mass of surfactants adsorbed [15]. As it results from the research and literature reports, surfactants are adsorbed on the electrode surface in a wide range of potentials. Excessively high concentration of such substances can obstruct the access to the electrode for diffusing zinc ions.

Adsorption of an organic compound on an electrode surface is considered as a replacement not only with water molecules but also with electrolyte ions $[6,16]$. Anionic surfactants such as SDS strongly adsorb over a wide range of potentials at the mercury electrode [17]. The formation of the dodecyl sulfate film characterized by multistep adsorption is directly connected with the electrode potential $[18,19]$. It was proposed that on hydrophobic surfaces such as gold [20,21], graphite [22], and mercury electrode [23], surfactants are adsorbed in the form of monolayer, hemicylindrical, or hemispherical structures. Below the $\mathrm{CMC}$, self-organizing aggregates are not formed in solution. In this case, individual molecules adsorb perpendicularly on solid surfaces forming a submonolayer or monolayer. Above the CMC, micelles adsorb and form multilayer on the surface $[6,24-26]$. In the longer time, the transformation into hemispherical surface micelles or bilayers of parallel adsorbed molecules occurs [26]. In the case of complete adsorption of films of sodium decyl and dodecyl sulfate, the shift of the point zero charge towards positive potentials has been proved [6].

The phenomenon of surfactant adsorption at different kinds of electrodes has been widely studied. However, the literature does not report on the electroreduction of these compounds. The aim of the present work focuses on the electrochemical reactions of sodium 2-ethylhexyl sulfate and its effect on the zinc electroreduction. In order to shift the overpotential of hydrogen evolution and to eliminate the UPD of zinc which occurs on the solid surface of an electrode $(\mathrm{Fe}, \mathrm{Au}, \mathrm{Pt})$, the mercury electrode was used in these studies.

\section{Experimental}

\section{Chemicals}

The reduction of sodium EHS salt was analyzed in the range of concentrations from $0.5 \times 10^{-3}$ to $8.0 \times 10^{-3} \mathrm{M}$. A voltammetric behavior of zinc $\left(1 \times 10^{-3} \mathrm{M}\right)$ was studied in solutions of EHS (from $1 \times 10^{-3}$ to $4 \times 10^{-3} \mathrm{M}$ ). The supporting electrolyte $\left(\mathrm{S}_{0}\right.$ ) containing $0.5 \mathrm{M} \mathrm{KCl}$ and $0.1 \mathrm{M} \mathrm{H}_{3} \mathrm{BO}_{3}$ was used for the preparation of all solutions. $\mathrm{pH}$ of medium was adjusted by $\mathrm{HCl}$ and $\mathrm{KOH}$. All compounds were purchased in SigmaAldrich. Solutions were prepared using analytical grade reagents and distilled water.

\section{Methods}

Electrochemical experiments were performed using the Autolab PGSTAT-20 potentiostat (Eco Chemie, Netherlands). A threeelectrode cell with a hanging mercury drop electrode (HMDE) (Metrohm 663 VA Stand model) as a working electrode (geometric area $3.93 \times 10^{-3} \mathrm{~cm}^{2}$ ), platinum as an auxiliary electrode, and a SCE as a reference electrode was applied in voltammetric analyses. The cyclic voltammetry (CV) measurements were carried out in the potential range from -0.1 to $-1.3 \mathrm{~V}$ vs. SCE. Before each voltammetric measurement, the solution was purged with argon for $20 \mathrm{~min}$ and an argon blanket was kept over the solution surface during the measurement. The $\mathrm{pH}$ of solutions was measured using a digital $\mathrm{pH}$ meter (CX-401 model, Elmetron, Poland).

The differential capacity analyses were performed using the same potentiostat equipped with a frequency response analyzer (FRA). The experiments were carried out in the potential range from -0.1 to $-1.3 \mathrm{~V}$ vs. SCE with the potential step of $0.01 \mathrm{~V}$ and the equilibration time of $5 \mathrm{~s}$. Each point of the measurement was performed with a new drop.

An electrochemical treatment of EHS was carried out in a cell with a volume of $160 \mathrm{~cm}^{3}$. A platinum plate was applied as a cathode and $\mathrm{TiO}_{2}(70 \%)-\mathrm{RuO}_{2}(30 \%) / \mathrm{Ti}$ as an anode with the geometric area of $20 \mathrm{~cm}^{2}$ each. Processes were performed in the range of current intensity from 0.05 to $0.3 \mathrm{~A}$ and process time from 10 to $180 \mathrm{~min}$. In order to assess a mineralization effect of EHS obtained in the electrochemical process, the total organic carbon (TOC) content was measured in the initial and post-reaction solutions using a Shimadzu TOC-5050A 
analyzer, in order to calculate mineralization $(\alpha \mathrm{TOC})$ according to the following equation:

$\alpha \mathrm{TOC}=\left(\frac{C_{0}-C}{C_{0}}\right) \times 100 \%$

where $\alpha \mathrm{TOC}$ is the TOC decrease (\%), $C_{0}$ is the organic carbon content in the initial solution $\left(\mathrm{mg} \mathrm{L}^{-1}\right)$, and $C$ is the organic carbon content in the solution after the reaction $\left(\mathrm{mg} \mathrm{L}^{-1}\right)$.

\section{Results and Discussion}

\section{The Influence of EHS on the Reaction of Zinc Electroreduction}

The effect of EHS addition on the zinc electroreduction process was studied by means of cyclic voltammetry at the mercury electrode (HMDE) in the solution of $\mathrm{S}_{0}+1 \times 10^{-3} \mathrm{M} \mathrm{ZnCl}_{2}$, $\mathrm{S}_{0}+4 \times 10^{-3} \mathrm{M}$ EHS, and their mixture $\left(\mathrm{S}_{0}+1 \times 10^{-3} \mathrm{M}\right.$ $\mathrm{ZnCl}_{2}+4 \times 10^{-3} \mathrm{M}$ EHS) with the scan rate of $0.2 \mathrm{~V} \mathrm{~s}^{-1}$. Figure 1 presents the cyclic voltammograms recorded in EHS solution $\left(\mathrm{S}_{0}+4 \times 10^{-3} \mathrm{M}\right.$ EHS $)$ and zinc solution in the presence and absence of the analyzed surfactant $\left(\mathrm{S}_{0}+1 \times 10^{-3} \mathrm{M} \mathrm{ZnCl}_{2}+\right.$ $4 \times 10^{-3} \mathrm{M} \mathrm{EHS}$ and $\mathrm{S}_{0}+1 \times 10^{-3} \mathrm{ZnCl}_{2}$ ). The currents recorded in the case of EHS are about 2 orders of magnitude less than the zinc reduction current. The whole $\mathrm{CV}$ curve of EHS reduction is shown in Fig. 2. The voltammetric reduction of $\mathrm{Zn}^{2+}$ ions occurs at $-1.04 \mathrm{~V}$ vs. SCE in the cathodic potential scan. The oxidation of reduced $\mathrm{Zn}^{2+}$ is observed during the reversed potential scan at $-0.98 \mathrm{~V}$ vs. SCE. The EHS addition to the zinc solution results in slight changes in the shape of the curves as well as the values of current density. A small shift of the zinc reduction peak about $0.014 \mathrm{~V}$ towards more negative potentials can be observed. This fact can suggests an insignificant inhibition of the zinc reduction at $-1.040 \mathrm{~V}$ vs. SCE, displacing it at $-1.054 \mathrm{~V}$ vs. SCE. Such

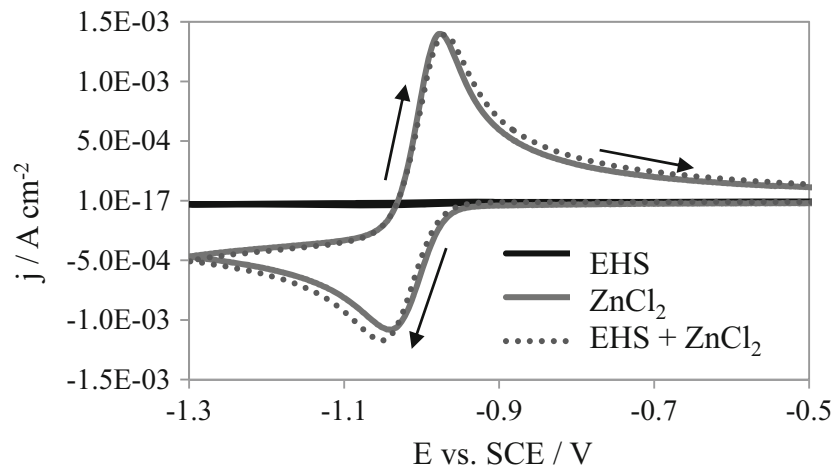

Fig. 1 Cyclic voltammograms recorded at the mercury electrode in solutions of $4 \times 10^{-3} \mathrm{M} \mathrm{EHS,} 1 \times 10^{-3} \mathrm{M} \mathrm{ZnCl}_{2}$, and their mixture $\left(4 \times 10^{-3} \mathrm{M} \mathrm{EHS}+1 \times 10^{-3} \mathrm{M} \mathrm{ZnCl}_{2}\right)$ in the supporting electrolyte at $v=0.2 \mathrm{~V} \mathrm{~s}^{-1}$

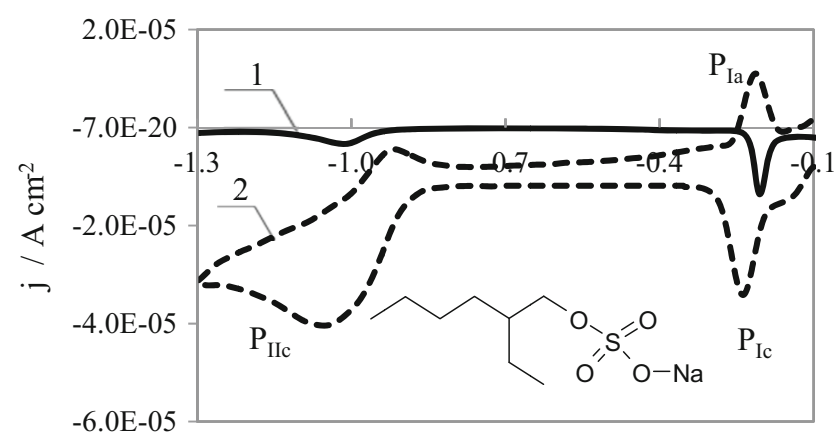

E vs. SCE / V

Fig. 2 Voltammograms of EHS electroreduction recorded at the mercury electrode in the solution of $2 \times 10^{-3} \mathrm{M}$ EHS in the supporting electrolyte; curve 1 differential pulse voltammogram, curve 2 cyclic voltammogram; $v=0.2 \mathrm{~V} \mathrm{~s}^{-1}$

results are usually interpreted as the adsorption of the substrate on the electrode surface and blockage of its active sites [27, 28]. An increase in the value of current density is connected with the additivity of the currents. The cyclic voltammogram of the EHS reduction shows the first adsorptive peak at $-0.23 \mathrm{~V}$ vs. SCE and the second well-shaped peak at $-1.06 \mathrm{~V}$ vs. SCE, wherein the recorded currents are much lower than those obtained for the zinc reduction.

\section{The Electrochemical Reaction of EHS}

The electrode reactions of EHS at the mercury electrode were studied by cyclic voltammetry and differential pulse voltammetry (DPV). If the peaks observed in the DPV curve characterize a diffusion-controlled process, their potentials correspond to the half-wave potentials, determined from $\mathrm{CV}$ curves. Both DPV and CV voltammograms (curves 1 and 2 in Fig. 2) show two electrode steps. Two peaks at -0.20 and $-1.01 \mathrm{~V}$ vs. SCE visible in the differential pulse voltammogram are connected with EHS reaction. Two pairs of peaks that are indicative of a two-state behavior are observed in the $\mathrm{CV}$ voltammogram registered in the EHS solution. A sharp peak, characteristic of an adsorption/desorption process, appears at the beginning of the negative potential scan at $-0.23 \mathrm{~V}$ vs. SCE, whereas the second peak is observed at $-1.06 \mathrm{~V}$ vs. SCE.

The scan rate effect on the EHS electroreduction was analyzed in the range from 0.01 to $0.9 \mathrm{~V} \mathrm{~s}^{-1}$ using cyclic voltammetry, and the results are presented in Fig. 3. Both anodic and cathodic peaks shift slightly in relation to the potential axis and become higher with the increasing value of the scan rate. The first $\left(\mathrm{P}_{\mathrm{Ic}}\right)$ and the second $\left(\mathrm{P}_{\text {IIc }}\right)$ peaks shift in the negative direction and become sharper. Such behavior points at an adsorption participation in the electrochemical process. Furthermore, the character of the current maxima in $\mathrm{P}_{\text {IIc }}$ seems to be complex, due to its separation with an increasing value of the scan rate (above $0.3 \mathrm{~V} \mathrm{~s}^{-1}$ ) in two better-shaped peaks. 


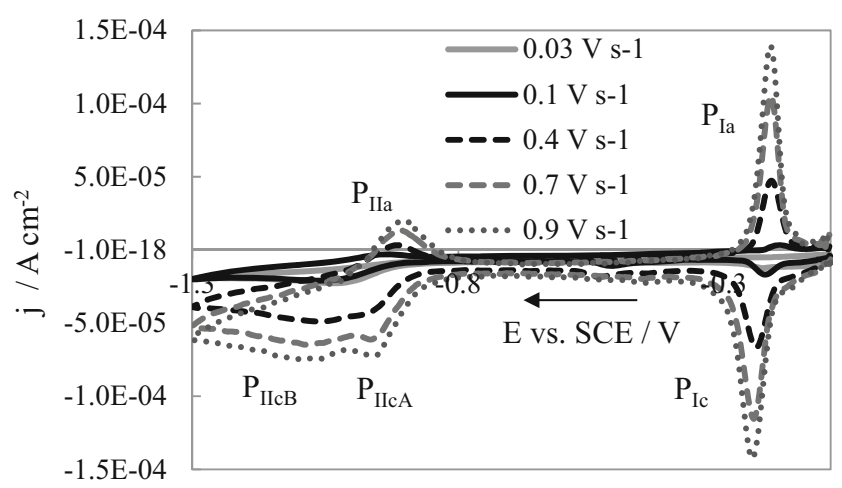

Fig. 3 Cyclic voltammograms recorded at the mercury electrode in solutions of $2 \times 10^{-3} \mathrm{M}$ EHS in the supporting electrolyte at various scan rates

Moreover, at the scan rate from 0.1 to $0.9 \mathrm{~V} \mathrm{~s}^{-1}$, an anodic $\mathrm{P}_{\text {III }}$ peak appears. This peak corresponds to the oxidation of the reduction product characterized by a $\mathrm{P}_{\text {IIcA }}$ peak.

In order to verify the nature of the reaction which is characterized by the peaks in $\mathrm{CV}$ voltammograms, the dependence of the cathodic peak current on the square root of the potential scan rate was determined for $\mathrm{P}_{\text {Ic }}$ and $\mathrm{P}_{\text {IIc }}$ peaks. The change in the current density, $j_{\mathrm{PIc}}$ Vs. $v^{1 / 2}$, is non-linear, which may indicate the participation of adsorption in the reaction. The dependence $j_{\text {PIc }}=f(v)$ (Fig. $4 \mathrm{a}$ ) points that the current density of the $\mathrm{P}_{\text {Ic }}$ peak is proportional to the scan rate. This means that the kinetics of the reaction characterized by $\mathrm{P}_{\mathrm{Ic}}$ is determined by adsorption. The shape and relative location of the $\mathrm{P}_{\text {Ic }}$ and $\mathrm{P}_{\text {Ia }}$ peaks also indicate the adsorptive nature of the process. The distance between these peak maxima varies from 0.022 to $0.034 \mathrm{~V}$ vs. SCE, depending on the polarization scan rate. The charge calculated by integration of the area under the $\mathrm{P}_{\text {Ic }}$ peak in the range of potentials from -0.1 to $-0.35 \mathrm{~V}$ vs. SCE is greater by $10 \%$ than the charge under the $\mathrm{P}_{\text {Ia }}$ peak. This means that the amount of adsorbed EHS is not desorbed entirely in the reversed cycle. The peak width at half height for a reversible adsorption process should be equal to $90.6 \mathrm{mV} / n$; thus, the number of exchanged electrons $(n)$ estimated from this value is 2 .

A linear dependence of the cathodic current density of $\mathrm{P}_{\text {IIc }}$ on the square root of the potential scan rate, $j_{\mathrm{PIIc}}=f\left(v^{1 / 2}\right)$, was also determined. Although this plot (Fig. 4b) is linear, it does not cross the origin of the axes which indicates that the second step of the EHS reduction is simultaneously controlled by diffusion and adsorption. This fact can suggest that the second step of EHS electroreduction is controlled by diffusion preceded by a chemical reaction. A dependence of $\log$ ip on $\log v$ (Fig. 4c) is linear with the slope of 0.4 , indicating a diffusion control of the electrode process. The slope close to 0.5 is expected for the diffusion-controlled process whereas 1.0 for the process controlled by the adsorption [29, 30].

The potential of the cathodic and anodic $\mathrm{P}_{\mathrm{I}}$ peaks changes insignificantly with the increase in the temperature (Fig. 5a),
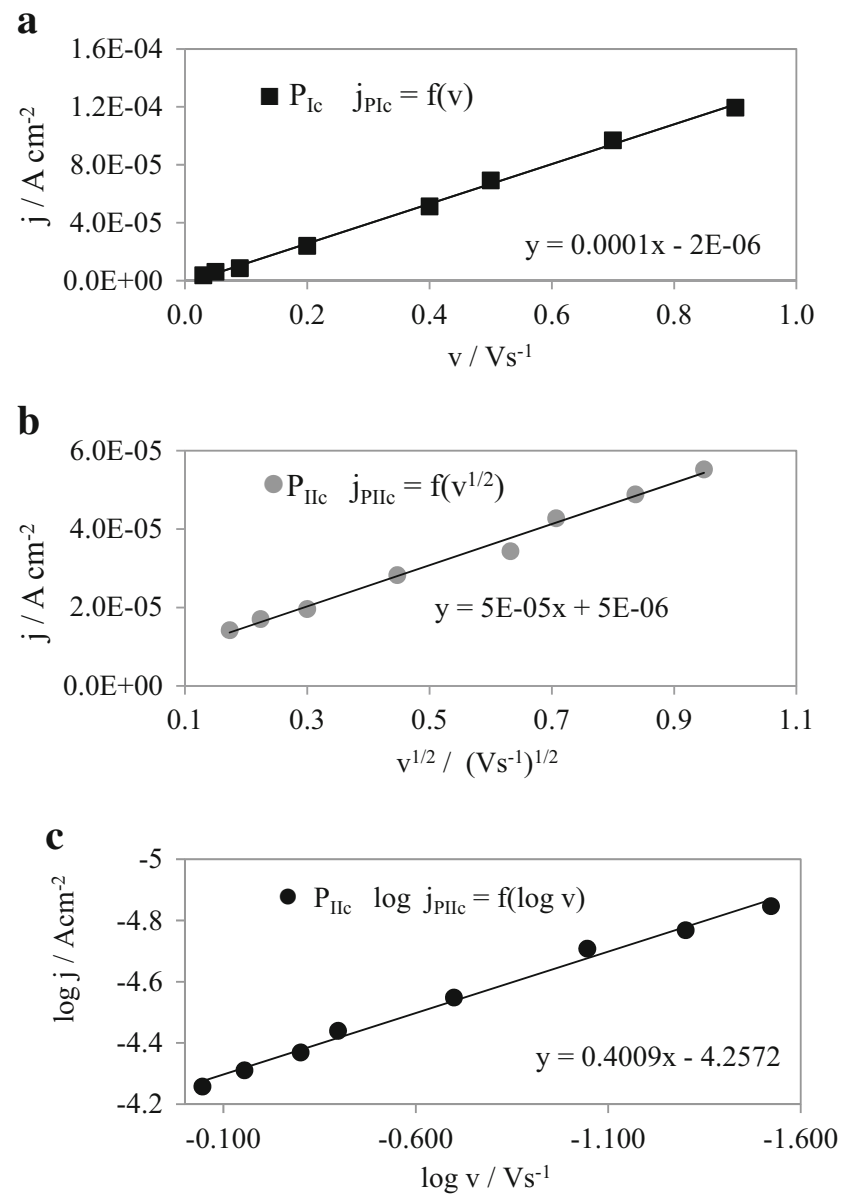

Fig. 4 Dependence of a the cathodic peak current density for PIc (black square) on the potential scan rate (v), $\mathbf{b}$ the cathodic peak current density for PIIcB (gray circle) on the square root of the potential scan rate $(v 1 / 2)$, and $\mathbf{c}$ the cathodic peak current density on the potential scan rate for PIIcB (black circle) in logarithm coordinates for the reduction of EHS at the $\mathrm{Hg}$ electrode

whereas the current density decreases (Fig. 5b). Such a drop in the current value confirms the adsorptive nature of the first peak [31].

The behavior of the $\mathrm{P}_{\text {IIc }}$ peak with the temperature is more complicated. As previously stated, the growth of the scan rate causes a separation of the $\mathrm{P}_{\text {IIc }}$ peak. Thus, the measurement carried out at $0.9 \mathrm{~V} \mathrm{~s}^{-1}$ results in the separation of $\mathrm{P}_{\text {IIc }}$ into two peaks at potentials -1.0 and $-1.1 \mathrm{~V}$ vs. SCE. The potentials of these two peaks practically do not change with the temperature. The first peak $\left(\mathrm{P}_{\text {IIcA }}\right)$ increases with the increasing temperature up to $30^{\circ} \mathrm{C}$ and then decreases and disappears, while the second peak $\left(\mathrm{P}_{\text {IIcB }}\right)$ increases with temperature all the time (Fig. 5c). Such behavior of the peak current indicates that the first of the separated peaks is controlled by adsorption while the second by diffusion [31].

The electrochemical reduction of EHS was studied in solutions with various $\mathrm{pH}$ values in the range from 3.0 to 8.0. The $\mathrm{d} E_{1 / 2} / \mathrm{dpH}$ dependence presented in Fig. 6 shows that $E_{1 / 2}$ practically does not depend on $\mathrm{pH}$. The $\mathrm{d} E / \mathrm{dpH}$ slopes are 
a

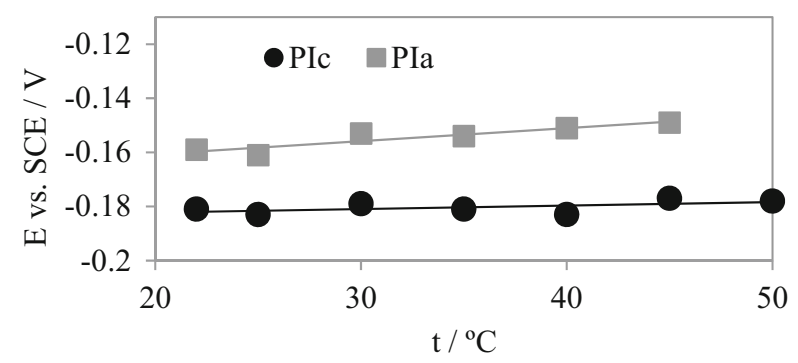

b

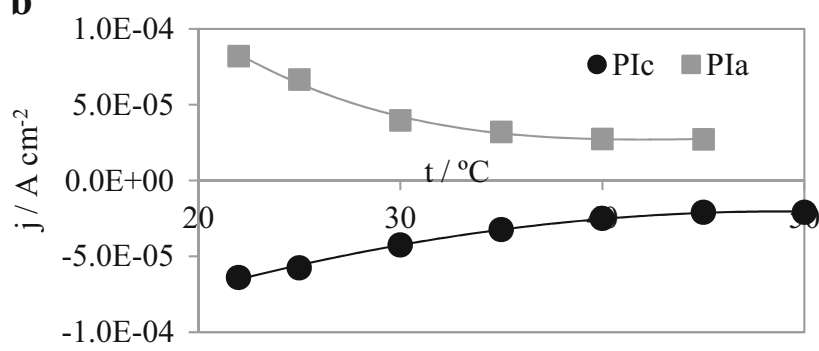

c

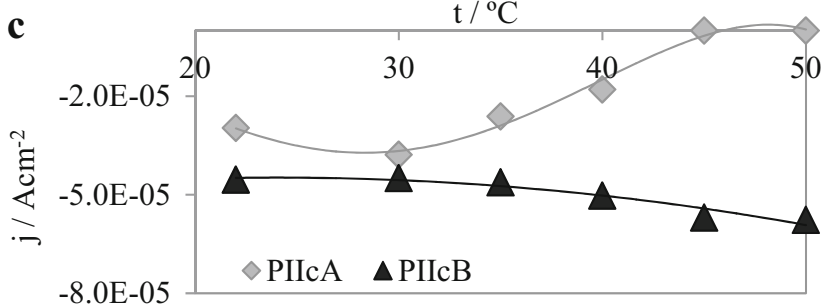

Fig. 5 Dependence of the a potential, b current density on the temperature $(t)$ for the cathodic $\mathrm{P}_{\mathrm{Ic}}$ (black circle) and anodic $\mathrm{P}_{\mathrm{Ia}}$ (gray square) peaks, and $\mathbf{c}$ current density on the temperature for the cathodic $\mathrm{P}_{\mathrm{IIcA}}$ (gray diamond) and $\mathrm{P}_{\mathrm{IIcB}}$ (black triangle) peaks

0.006 and 0.007 for cathodic $\mathrm{P}_{\mathrm{Ic}}$ and $\mathrm{P}_{\mathrm{IIc}}$ peaks, respectively, suggesting the lack of $\mathrm{pH}$ effect on the first and second steps of the EHS reduction.

\section{Adsorption of EHS on Mercury Electrode}

It is well known that the surfactant properties change above a critical micelle concentration (CMC). The $\mathrm{CMC}$ value can be determined on the basis of changes in conductivity due to changes in surfactant concentration [32-34]. The CMC value

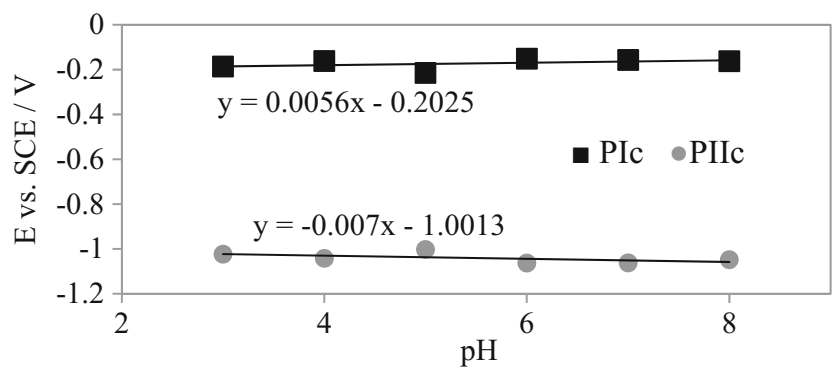

Fig. 6 The effect of $\mathrm{pH}$ on the half-wave $\left(E_{1 / 2}\right)$ potential for $\mathrm{P}_{\mathrm{Ic}}$ (black square) and $\mathrm{P}_{\text {IIc }}$ (gray circle) peaks of EHS reduction determined for EHS in the supporting electrolyte $\left(\mathrm{S}_{0}\right)$ on the basis of electrical conductivity changes is $4.6 \times 10^{-3} \mathrm{M}$.

A contribution of adsorption to the electrochemical reduction reaction of EHS may be analyzed by the measurements of electrical double-layer capacitance. In order to determine the potential range of the EHS adsorption at the mercury electrode surface, impedance analysis was carried out. Measurements were started by checking the effect of frequency on the differential capacity of the double layers in the presence of the surfactant. Lowering the frequency causes an increase in the differential capacity of adsorption-desorption peaks. A frequency of $24.64 \mathrm{~Hz}$ was used for further measurements.

Figure 7 presents the differential capacity curves recorded in the supporting electrolyte $\left(\mathrm{S}_{0}\right)$ and $0.76,4.0$, and $6.0 \times 10^{-3} \mathrm{M}$ solutions of EHS. The differential capacity curve for the solution of $\mathrm{S}_{0}$ is included in the panel for comparative purposes. The data show that the addition of surfactant to the supporting electrolyte causes a decrease in the double-layer capacitance, pointing to the EHS adsorption on the electrode surface. Two adsorptive-desorptive peaks separated by a long flat section are clearly observed. The curves for different EHS concentrations are similar in shape. The major difference with the increase in concentration comprises the size of the peaks and the extension of the potential range of adsorption. For $c=4 \times 10^{-3} \mathrm{M}$, the coverage on the mercury surface is maximum in the potential range -0.4 to $-0.8 \mathrm{~V}$ vs. SCE, reaching a differential capacity value equal to $3.18 \times 10^{-6} \mathrm{~F} \mathrm{~cm}^{-2}$. The potential at which the electrical double layer collapses and the electrode surface has no excess charge is known as the potential of zero charge (PZC), and it corresponds to the potential at which the differential capacity $\left(C_{\text {diff }}\right)$ reaches its minimum. The potential of zero charge, at which adsorption is the greatest, was determined by integration of the differential capacity curves reaching the value of $-0.56 \mathrm{~V}$ vs. SCE for $0.76 \times 10^{-3} \mathrm{M}$ and $-0.59 \mathrm{~V}$ vs. SCE for $6.0 \times 10^{-3} \mathrm{M}$. Such a shift of PZC for complete coverage of the surface by the EHS may indicate the formation of a condensed film, in which specifically adsorbed sodium and potassium ions control the shift [6].

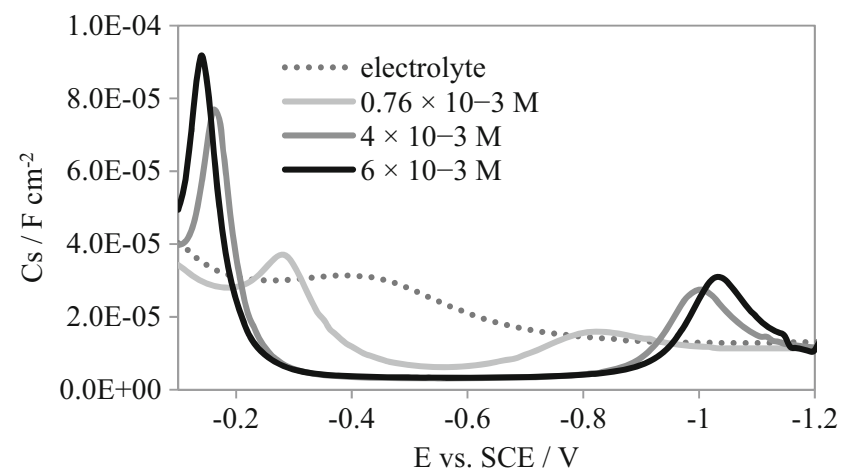

Fig. 7 Differential capacity-potential dependences recorded in the supporting electrolyte $\left(\mathrm{S}_{0}\right)$ and EHS solutions in the concentration range from 0.76 to $6.0 \times 10^{-3} \mathrm{M}$ at the mercury electrode for $24.64 \mathrm{~Hz}$ 
At each curve presented in Fig. 7, two peaks are observed. The first peak corresponding to the EHS adsorption-desorption depends on the concentration and varies from -0.14 to $-0.28 \mathrm{~V}$ vs. SCE. At the most negative potentials, the differential capacity curves for EHS merge with the curve recorded for the electrolyte, indicating the desorption of the surfactant from the electrode surface.

The differential capacity curves recorded in the positive and negative directions show hysteresis. The change of the potential scan direction from negative to positive leads to a shift in potentials of adsorptive-desorptive peaks at about $0.1 \mathrm{~V}$ towards positive potentials. This may indicate a slow adsorption/desorption process, suggesting a differential capacity in a long term of measurement. The formation of the condensed film also requires some time. The dependence of the differential capacity on time is presented in Fig. 8.

It was found that the adsorption of a similar anionic surfactant such as sodium dodecyl sulfate (SDS) depends on the applied potential [35]. Therefore, the effect of potential on differential capacity-time transients registered at various potentials in the solution of EHS at concentrations below and above CMC $\left(0.76 \times 10^{-3}\right.$ and $\left.6.00 \times 10^{-3} \mathrm{M}\right)$ was studied. A slow decrease in the differential capacity in the case of $0.76 \times 10^{-3} \mathrm{M}$ of EHS (Fig. 8a) signifies the adsorption of the surfactant on the mercury surface. The increase in the differential capacity is connected with the desorption of molecules from the electrode, which may indicate the reversibility of the adsorption process [36, 37]. The slight increase in capacity, indicating a partial desorption of EHS, is observed in the potential range from -0.46 to $-0.86 \mathrm{~V}$
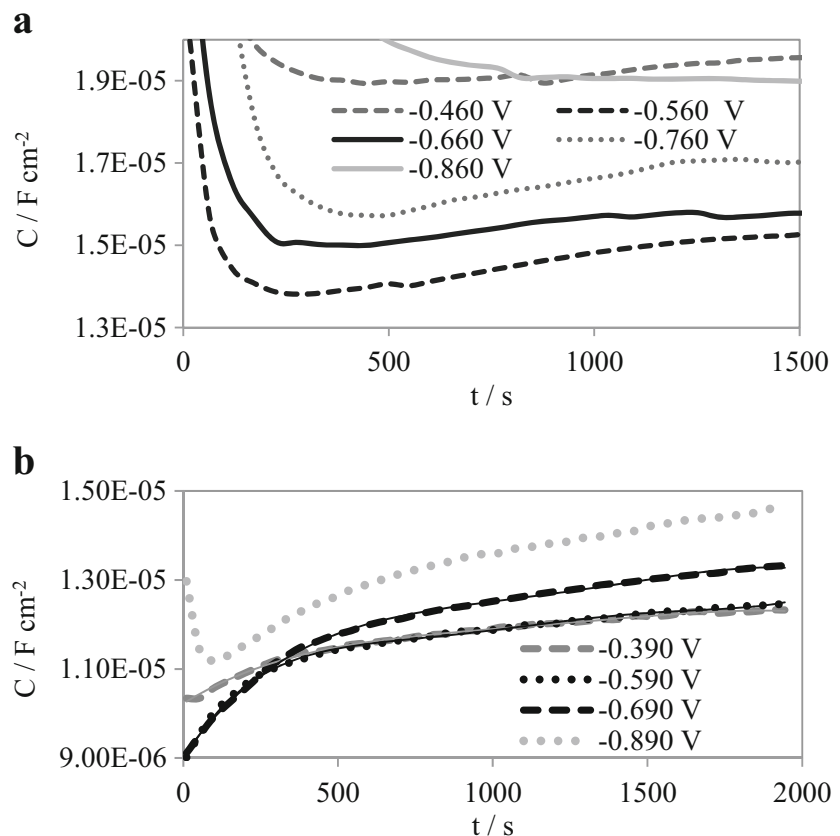

Fig. 8 Time dependence of the differential capacity of a $0.76 \times 10^{-3} \mathrm{M}$ EHS and b $6.00 \times 10^{-3} \mathrm{M}$ EHS at various potentials. Symbols experimental values; solid lines theoretical values calculated from Eq. (1) vs. SCE. The dynamics of adsorption is very small, which may suggest a change of the adsorption character. A typical irreversible adsorption was observed at each potential outside the above range.

The formation of the molecular film on the mercury electrode requires a sufficient concentration of surfactant molecules in the bulk as well as at the electrode surface. Thus, differential capacity-time curves depending on the applied potential were also recorded for EHS with a concentration above the $\mathrm{CMC}$, i.e., $6 \times 10^{-3} \mathrm{M}$ (Fig. $8 \mathrm{~b}$ ). Already at the start of registration, the differential capacity reaches its minimum $\left(C_{\mathrm{min}}\right)$ and then rises to the equilibrium value $\left(C_{\mathrm{eq}}\right)$, which is an established value of $C_{\text {diff }}$ registered in a long term. It characterizes an equilibrium between the single surfactant molecules and a hemimicelle formation $[6,19,23,26]$. The reason for the differential capacity increase in a long term is a probable impediment of the access to the electrode by forming micelles and thus the hindrance of adsorption. The decrease in the applied potential causes a decrease in the initial capacity, reaching its minimum at PZC, i.e., $-0.59 \mathrm{~V}$ vs. SCE. A subsequent decrease in the potential results in an increase in the initial value of differential capacity. The character of the changes in the range of potentials from -0.39 to $-0.79 \mathrm{~V}$ vs. $\mathrm{SCE}$ is similar and can be described by kinetic Eqs. (2) and (3) of the first-order surface reaction [26].

$C=\Theta\left(C_{\mathrm{eq}}-C_{\min }\right)+C_{\min }$

$\Theta=1-\exp (-k t)$

where $\Theta$ is the coverage degree; $C, C_{\min }$, and $C_{\text {eq }}$ are the temporary, minimal, and equilibrium capacities, respectively; $k$ is the rate constant; and $t$ is the time.

The effect of temperature on the differential capacity-time transients was also analyzed, and the obtained curves are shown in Fig. 9. Measurements were carried out for the $6.00 \times 10^{-3} \mathrm{M}$ EHS system at a constant potential of zero

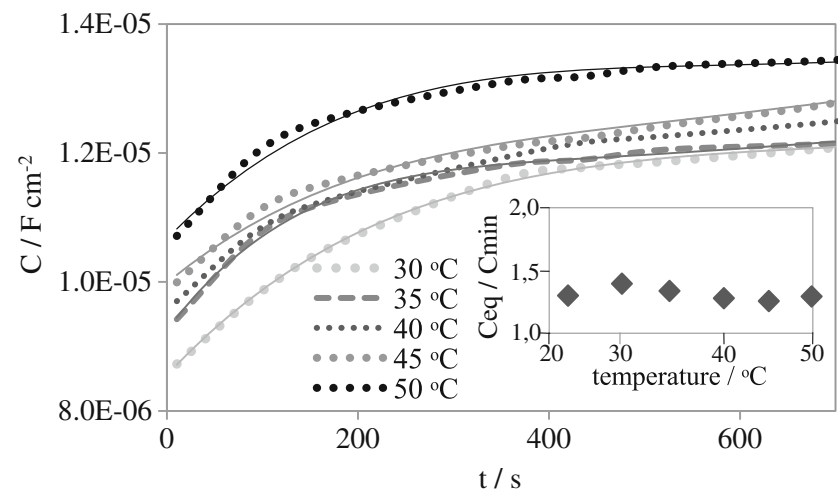

Fig. 9 Time dependence of differential capacity of $6.00 \times 10^{-3} \mathrm{M}$ EHS at various temperatures and at potential of zero charge $(-0.590 \mathrm{~V}$ vs. SCE). Symbols experimental values; solid lines theoretical values calculated from Eq. (1). Inset temperature dependence of $C_{\mathrm{eq}} / C_{\mathrm{min}}$ 
charge $(-0.59 \mathrm{~V}$ vs. SCE), corresponding to the maximum degree of electrode coverage. The obtained curves show that the differential capacity in a long term depends on and slightly increases with the temperature. These curves were also modeled using Eq. (2), giving a good theoretical description of the experimental data. The $k$ parameter calculated from Eqs. (2) and (3) also slightly increases with temperature and is of the order $4.6 \times 10^{-3}$. The $C_{\mathrm{eq}} / C_{\min }$ ratio indicates whether the process involves the formation of micelles in the form of hemispherical or hemicylindrical [38]. The temperature dependence of the ratio $C_{\mathrm{eq}} / C_{\mathrm{min}}$ (inset in Fig. 9) is practically constant in the range of the analyzed temperatures and amounts to $1.35 \pm 0.06$. This value approaches 1.27 predicted by the theory in the case of the formation of hemicylindrical surface micelles [36].

The adsorption potential range defined for a particular surfactant can be compared between different electrode materials, taking into account the PZC [18]. A direct comparison between the EHS adsorptions on different electrodes can be made by plotting the corresponding capacity curves on a rational potential $\left(E_{\text {rational }}\right)$ calculated as follows:

$E_{\text {rational }}=E-\mathrm{PZC}$

where $E$ is the potential measured, $\mathrm{PZC}$ is the potential of zero charge, and $E_{\text {rational }}$ is the rational potential.

The morphology of surfactants at electrode interfaces depends mainly on the electrical state of a surface (surface charge density or the electrode potential), concentration, and the type of surfactant, e.g., anionic (SDS), cationic ( $N$-decyl- $N, N, N$-trimethylammonium triflate, DeTATf), and zwitterionic ( $N$-dodecyl- $N, N$-dimethyl-3ammonio-1-propanesulfonate, DDAPS) [39]. The surface concentrations for DeTATf are lower than those for DDAPS and SDS and much lower than the maximum coverage expected for a monolayer of vertically adsorbed molecules.

In order to determine the coverage of the electrode by the surfactant, the charge defined as the area under the adsorption peak $\left(\mathrm{P}_{\mathrm{Ic}}\right)$ in the $\mathrm{CV}$ voltammogram was calculated. The number of adsorbing surfactant molecules for different concentrations and scan rates (the surface concentration) was related to the geometric area of the mercury electrode equal to $3.93 \times 10^{-3} \mathrm{~cm}^{2}$. The change in the EHS concentration from $0.76 \times 10^{-3}$ to $2.00 \times 10^{-3} \mathrm{M}$ results in the growth of coverage degree, while a further increase practically does not cause any change. The degree of coverage in the case of $2.00 \times 10^{-3} \mathrm{M}$ of EHS is $2.414 \times 10^{13}$ and $2.95 \times 10^{13}$ particles $\mathrm{cm}^{-2}$ for the scan rates lower and higher than $0.45 \mathrm{~V} \mathrm{~s}^{-1}$, respectively.

In order to predict how the EHS adsorbs on the mercury surface, the quantum-chemical calculations were done using the WinMopac 97 program [40]. The effect of the solvent (water) was considered using the COSMO method. On the basis of band structure calculations and experimental studies, the fcc (100) structure is considered the best model of liquid mercury [41, 42]. Therefore, this model was applied in the calculations. First, geometries of the mercury cluster and the EHS molecule were optimized separately using semi-empirical PM3 Hamiltonian. Distances between mercury atoms of the optimized cluster at an external polarization of $0.0 \mu \mathrm{C}$ amount to $2.93 \AA$. Subsequently, both systems were merged by bringing them closer and creating a bond between oxygen and mercury (Fig. 10). Consequently, the energy of the whole system diminishes, indicating the formation of a more stable form. Furthermore, the charge transfer from the EHS molecule on the mercury cluster is observed, confirming the formation of a chemical bond with the length of $2.06 \AA$. The slope of the EHS particle to the mercury surface is $77^{\circ}$.

The performed calculations show that the surfactant strongly adsorbs on the mercury surface. A single EHS molecule theoretically covers the surface equal to $3.85 \times 10^{-15} \mathrm{~cm}^{2}$ $\left(28.26 \AA^{2}\right)$ calculated for a radius of a cross section equal to $3 \AA$, which includes the head of the sulfonate group in the solvated form. The mercury electrode with a surface of $3.93 \times 10^{-3} \mathrm{~cm}^{2}$ can be covered by $3.53 \times 10^{14}$ of EHS molecules per square centimeter oriented in the manner ensuring the maximum degree of coverage. The value of the surface coverage calculated in this way is about 1 order of magnitude larger than that calculated from the integration of the area under the CV curve.

\section{Electrochemical Mineralization}

As we showed previously, another additive to the electroplating bath - benzylideneacetone (BDA) - is mineralized on both the zinc and the platinum electrodes [28]. In order to determine the EHS mineralization in electrochemical process, an electrode with a low overpotential for hydrogen evolution, i.e., platinum electrode, was used.

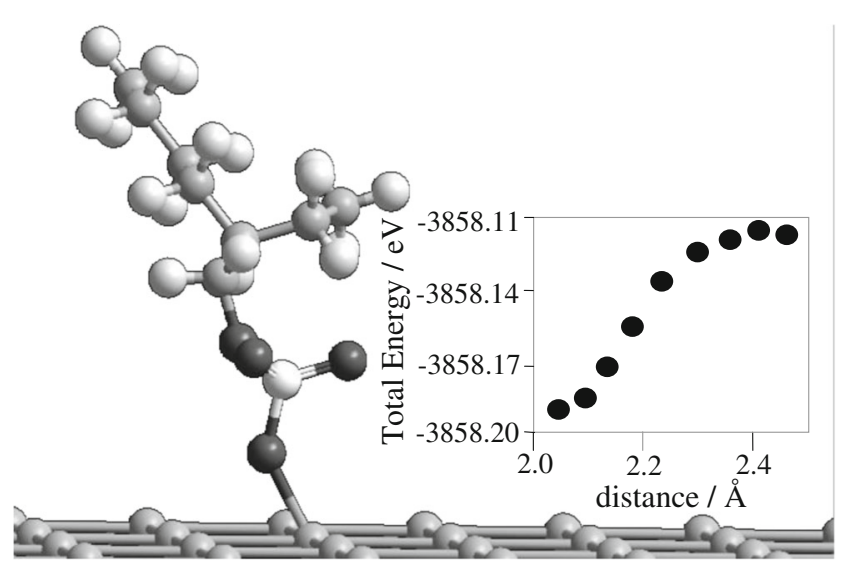

Fig. 10 A model of the adsorbed EHS molecule on the mercury surface. Inset effect of a distance between the mercury cluster and the EHS molecule on the total energy of the system 


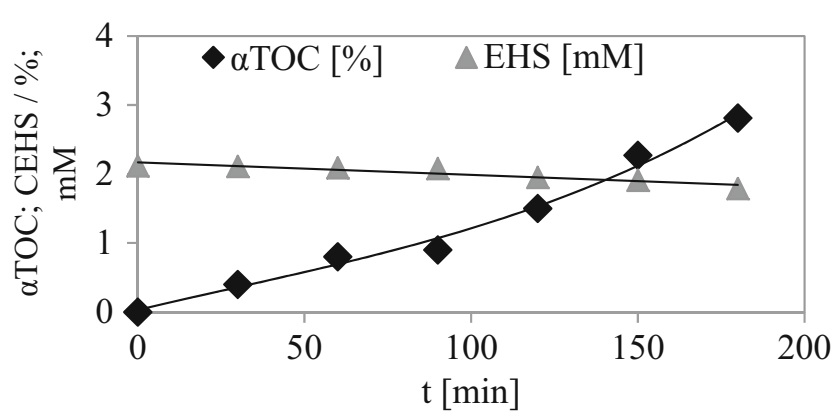

Fig. 11 Dependence of mineralization ( $\alpha \mathrm{TOC}$ ) (black circle) and concentration of EHS (gray triangle) on reaction time $(t)$ at $0.3 \mathrm{~A}$ of electrolysis

Literature reports do not point to what extent EHS is destroyed under the zinc electroplating conditions. To investigate the quantitative destruction, the electrochemical processes were carried out. A platinum cathode and a $\mathrm{TiO}_{2}$ $(70 \%)-\mathrm{RuO}_{2}(30 \%) / \mathrm{Ti}$ anode were used in the processes. Total organic carbon was analyzed to determine a decrease in the organic substance content defined as mineralization $\left(\alpha\right.$ TOC, Eq. (1)). The initial solution of EHS $\left(2 \times 10^{-3} \mathrm{M}\right)$ and solutions after electrochemical reactions were analyzed. According to a $\alpha \mathrm{TOC}=f(t)$ dependence presented in Fig. 11, only a very small mineralization degree $(3 \%)$ is observed. Thus, one can conclude that the surfactant practically does not undergo mineralization during the 180-min electrolysis. However, a more significant decrease in EHS concentration from 2.12 to $1.79 \mathrm{mM}$, i.e., $15 \%$, was found by ion chromatography measurements which suggest a part decomposition of EHS to another organic compounds.

\section{Conclusions}

The behavior of sodium 2-ethylhexyl sulfate in the electrochemical reactions and its effect on zinc electroreduction were investigated at the mercury electrode. The addition of surfactant to the $\mathrm{Zn}^{2+}$-containing solution results in a slight shift of the zinc evolution potential and a small increase in the current reduction. EHS undergoes a two-step reduction, where the first step is controlled by adsorption while the second by simultaneous adsorption and diffusion. In the analyzed $\mathrm{pH}$ range from 3 to 8 , EHS electroreduction practically does not depend on the reaction medium.

Differential capacity-potential measurements indicate a strong adsorption on the electrode surface in a wide potential range, which depends on the EHS concentration. The capacity registered in a long term shows some reversibility of the adsorption in the potential range from -0.46 to $-0.86 \mathrm{~V}$ vs. SCE at surfactant concentrations below the CMC. However, if the concentration is higher than the $\mathrm{CMC}$, an increase in differential capacity indicating the formation of hemicylindrical surface micelles is observed. The calculated $C_{\mathrm{eq}} / C_{\min }$ ratio amounts to $1.35 \pm 0.06$.

The 180-min electrolysis at the current 0.3 A leads to only $3 \%$ mineralization of EHS, whereas the degree of its decomposition reaches $15 \%$.

Open Access This article is distributed under the terms of the Creative Commons Attribution 4.0 International License (http:// creativecommons.org/licenses/by/4.0/), which permits unrestricted use, distribution, and reproduction in any medium, provided you give appropriate credit to the original author(s) and the source, provide a link to the Creative Commons license, and indicate if changes were made.

\section{References}

1. M.C. Li, L.L. Jiang, W.Q. Zhang, Y.H. Qian, S.Z. Luo, J.N. Shen, J. Solid State Electrochem. 11, 549 (2007)

2. D. Sylla, C. Rebere, M. Gadouleau, C. Savall, J. Creus, P. Refait, J. Appl. Electrochem. 35, 1133 (2005)

3. A. Gomes, M.I. da Silva Pereira, Electrochim. Acta 51, 1342 (2006)

4. B.C. Tripathy, S.C. Das, G.T. Hefter, P. Singh, J. Appl. Electrochem. 27, 673 (1997)

5. L. Onciu, L. Muresan, J. Appl. Electrochem. 21, 565 (1991)

6. U. Retter, M. Tchachnikova, J. Electroanal. Chem. 550-551, 201 (2003)

7. C. Tanford, The hydrophobic effect: formation of micelles and biological membranes, second edn. (Wiley-Interscience, New York, 1980), p. 233

8. D. Myers, Surfaces, interfaces, and colloids: principles and applications, second edition (John Wiley \& Sons, Inc.VCH, New York, 1999), pp. 384-387

9. C.-E. Lin, J. Chromatogr. A 1037, 467 (2004)

10. J. Nieszporek, S. Afr, J. Chem. 67, 1 (2014)

11. A. Aramata, S. Terui, S. Taguchi, T. Kawaguchi, K. Shimazu, Electrochim. Acta 41(5), 761 (1996)

12. M.A. Quaiyyum, A. Aramata, S. Moniwa, S. Taguchi, M. Enyo, J. Electroanal. Chem. 373(1-2), 61 (1994)

13. A. Gomes, M.I. da Silva Pereira, Electrochim. Acta 52, 863 (2006)

14. C. Cachet, R. Wiart, J. Electrochem. Soc. 141, 131 (1994)

15. L. Mivehi, R. Bordes, K. Holmberg, Langmuir 27, 7549 (2011)

16. S. Trasatti, Electrochim. Acta 37, 2137 (1992)

17. T. Wandlowski, M. Hromadova, R. de Levie, Langmuir 13, 2766 (1997)

18. M. Chen, I. Burgess, J. Lipkowski, Surf. Sci. 603, 1878 (2009)

19. A. Avranas, A. Komnianou, U. Retter, J. Colloid Interf. Sci. 264, 407 (2003)

20. I. Burgess, C.A. Jeffrey, X. Cai, G. Szymanski, Z. Galus, J. Lipkowski, Langmuir 15, 2607 (1999)

21. H.C. Schniepp, D.A. Saville, I.A. Aksay, Langmuir 24, 626 (2008)

22. W.A. Ducker, L.M. Grant, J. Phys. Chem. 100(28), 11057 (1996)

23. U. Retter, A. Avranas, Langmuir 17, 5039 (2001)

24. Y.L. Chen, S. Chen, C. Frank, J. Israelachvili, J. Colloid Interface Sci. 153, 244 (1992)

25. M.W. Rutland, J.L. Parker, Langmuir 10, 1110 (1994)

26. U. Retter, M. Tchachnikova, A. Avranas, J. Colloid Interf. Sci. 251, 94 (2002)

27. H. Yan, J. Downes, P.J. Boden, S.J. Harris, J. Electrochem. Soc. 143(5), 1577 (1996)

28. A. Perek-Dlugosz, A. Socha, L. Osiewala-Just, J. Rynkowski, K. Dziewulska-Czubak, Electrochim. Acta 123, 412 (2014) 
29. A.J. Bard, L.R. Faulkner, Electrochemical methods, fundamentals and applications, 2nd ed., John Wiley \& Sons, New York, 2001, pp. 236, 503, 709

30. J.A. Harrison, Z.A. Khan, J Electroanal Chem Interfacial Electrochem 28(1), 131 (1970)

31. A. Zolfaghari, G. Jerkiewicz, J. Electroanal. Chem. 420, 11 (1997)

32. K.D. Danov, P.A. Kralchevsky, K.P. Ananthapadmanabhan, Adv. Colloid Interfac. 206, 17 (2014)

33. N. Jalšenjak, Đ. Težak, Chem. Eur. J. 10, 5000 (2004)

34. M. Pérez-Rodríguez, L.M. Varela, M. García, V. Mosquera, F. Sarmietno, J. Chem. Eng. Data 44, 944 (1999)
35. H. Sawamoto, Nippon Kagaku Kaishi 7, 511 (1998)

36. H. Sawamoto, K. Gamoh, J. Electroanal. Chem. 283(1-2), 421 (1990)

37. H. Sawamoto, Anal. Sci. 19, 1381 (2003)

38. U. Retter, Langmuir 16, 7752 (2000)

39. I. Burgess, V. Zamlynny, G. Szymanski, J. Lipkowski, J. Majewski, G. Smith, S. Satija, R. Ivkov, Langmuir 17(11), 3355 (2001)

40. J. J. P. Stewart, MOPAC ver. 2.0 manual, (Fujitsu Limited, Tokyo, Japan, 1998)

41. L.F. Mattheiss, W.W. Warren, Phys. Rev. B 16, 624 (1977)

42. Y. Waseda, K. Suzuki, Phys. Status Solidi C 40(1), 183 (1970) 